The Egyptian Journal of Hospital Medicine (October 2018) Vol. 73(1), Page 5896-5906

\title{
Internal Limiting Membrane Peeling for Diffuse Tractional Diabetic Macular Oedema Mohamed I. EL-Kasaby
}

Department of Ophthalmology, Faculty of Medicine for girls, Al-Azhar University, Cairo- Egypt. Corresponding author: Mohamed EL-Kasaby, email: Ammarelkasaby@yahoo.com., Mobile: +2 01001241403 010423513- Fax:+202:22731988.

\begin{abstract}
Aim: To determine the efficacy of internal limiting membrane (ILM) peeling vitrectomy for diffuse tractional diabetic macular oedema. Patients and methods: A prospective non -randomized interventional study was carried out at Nour- EL-Hayaha Eye Center (Cairo) between March, 2015 and March, 2017. 40 eyes of 31 patients with symptomatic marked diminution of vision and tractional diabetic macular oedema were enrolled in this study. Ocular examinations included measurements of best corrected visual acuity (BCVA) at a distance using a logarithm of the minimum angle of resolution (logMAR) scale, refractive status using an autorefractometer (KR-8100; Topcon corporation, Tokyo, Japan), IOP was measured by Goldman applanation tonometry (CT-80; Topcon corporation, Tokyo, Japan), and fundus evaluation using an indirect ophthalmoscope were obtained. 23-gauge vitrectomy with internal limiting membrane peeling assisted by staining by brilliant blue $\mathrm{G}$ stain(BBG) were performed for all patients with a follow -up period at least 6 months. Spectral domain optical coherent tomography (SD OCT) images were obtained at the follow -up visits to determine the presence of an epiretinal membrane (ERM). Results: At 6 months there was a median $200 \mu$ decrease from baseline in the central subfield thickness $(\mathrm{P}<0.005)$. Mean change in the central subfield macular thickness was $-150 \mu$ ( $\mathrm{SD} \pm$ 130.56). There was 7 (17.5\%) cases developed iatrogenic retinal break during removal of posterior vitreous and treated by endodiathermy, of greatest importance, $3(7.5 \%)$ eyes developed a vitreous hemorrhage treated by conservative treatment after B scan evaluation, and $1(2.5 \%)$ eye developed a retinal detachment. All complications were successfully managed. 23 out of 40 eyes $(57.5 \%)$ underwent cataract surgery with IOL implantation within 6 months of pars plana vitrectomy. Transient elevation of IOP was developed in 7(17.5\%) eyes and managed carefully with antiglucomatous drugs. One eye developed persistent elevation of IOP that required maintenance anti glaucomatous drug. Also macular hole developed in one eye and epiretinal membrane developed in one eye. Conclusion: ILM peeling achieved higher anatomic success with a reduced need for additional surgical interventions and or event postoperative ERM formation that might result in subsequent visual loss.
\end{abstract}

Keywords: Internal limiting membrane. tractional diffuse macular oedema .brilliant blue G.

\section{Introduction}

The internal limiting membrane (ILM) is a very thin and transparent acellular membrane on the surface of the retina. It is adjacent to Muller cell foot plates (lamina rara externa $(0.03-0.06 \mu)$. The lamina densa is thinnest at the fovea and thicker in the posterior pole than at the equator or vitreous base; it plays an important role in the early stages of retinal development; however, its function in adults is not yet understood. ${ }^{(1),(2)}$ Because the ILM rests on the retinal nerve fiber layer (RNF)ganglion cell layer (GCL)complex ,it can theoretically damage these layers during the process of peeling with subsequent significant bearing on postoperative visual acuity ${ }^{(3)}$. Diabetic macular oedema (DME), caused by intraretinal fluid accumulation in the macula, is the most common cause of visual impairment in diabetic patients. The pathogenesis is multifactorial and includes breakdown of the blood-retinal barrier (BRB) secondary to weakened capillary intercellular tight junctions, loss of pericytes, and leukostasis in the retinal vessels and vasoactive factors such as vascular endothelial growth factorA (VEGF-A), various growth factors, and matrix metalloproteinases. Abnormalities at the vitreoretinal interface (the posterior vitreous cortex and ILM) have also been found to promote DME. Specifically, the hyaloid becomes taut and thickened with induced cellular proliferation and production of cytokines. The fovea and the vitreous base are the points at which the posterior vitreous cortex and the ILM have the strongest attachment. Advanced Glycation End-Products (AGEs), accumulated in the posterior vitreous cortex, increase cross-linking of collagen fibrils and induce structural changes in the posterior hyaloid that strengthen vitreomacular adhesions 
between the posterior hyaloid and ILM. This is further aggravated by AGE receptors (RAGEs), which are attached to the footplates of the Müller cells and extend to the external limiting membrane (ELM). RAGE activation by the binding of AGEs stimulates VEGF up regulation and retinal vessel permeability, further exacerbating $\mathrm{DME}^{(4)}$.

\section{Patients and methods:}

A prospective non -randomized interventional study was carried out at Nour- EL-Hayaha Eye Center (Cairo) between March, 2015 and 2017. 40 eyes of 31 patients with symptomatic marked diminution of vision and tractional diabetic macular oedema were enrolled in this study. The study protocol was adhered to the tenets of declaration of Helisinki and was approved by the ethics board of AL- Azhar university .An informed written consent was taken from each participant in the study after a detailed explanation of the procedure and possible benefits and risks. A full history included medical history (duration of diabetes, controlled or not) other relative systemic diseases and family history of diabetes .Glycosylated hemoglobin were controlled in this study. A detailed clinical history that included the onset of visual disturbances and history of ocular diseases was obtained at the initial visit at which tractional diabetic macular oedema was detected. All patients underwent a full ocular examination included measurement of decimal visual acuity,slit lamp examination and indirect ophthalmoscopy. Pre -interventional fundus fluorescein angiography using Topcon TRC 501X retinal camera, Spectral domain optical coherent tomography ( SD OCT) to document macular thickness, configuration of tractional macular oedema, vitreomacular traction and an taut posterior hyaloid. The exclusion criteria were lens opacity precluding OCT, massive hard exudates in the fovea, only focal macular oedema ,history of previous vitreoretinal surgery coexisting ocular diseases other than tractional diabetic macular, macular ischaemia and monocular patient. Patients with a history of previous ocular surgery and coexisting ocular diseases other than tractional diabetic macular oedema were excluded. Patients who failed to complete follow-up examinations one month after operation were also excluded. All patients underwent pars plana vitrectomy (PPV) for tractional diabetic macular oedema, sutureless 23gauge by a single surgeon. A follow -up period of at least 6 months after the 23 gauge vitrectomy, SD OCT images were obtained at the follow -up visits to determine the presence of an epiretinal membrane (ERM).

\section{Surgical procedure}

All the cases were prepared and draped in the standard fashion. Surgery was performed under local anesthesia with peribulbar block. Seclerotomy was done in two-step entry for 23gauge vitrectomy. A 23-gauge micro vitreoretinal blade trocar (MVR) blade was introduced into the conjunctiva-sclera in a beveled direction to create a 10 to 20 degrees angled incision and to obtain tunnels parallel to the corneoscleral limbus. The tunnel incision created in this fashion was left without a suture at the end of the operation (Fig.1) (18). Subsequent intraoperative assessment included careful documentation of any surgical steps, instrumentation, and the gas used as the tamponading agent. All patients had undergone 3 port vitrectomy using Alcon Accurus (pneumatic vitreous cutter). Non-contact wide angle viewing system (BIOM) was used for visualization. Illumination was provided by xenon light, through 23 -guage light pipe. The vacuum was set at 100 $\mathrm{mmHg}$ to $400 \mathrm{mmHg}$ with bottle height of 50 $150 \mathrm{~cm}$. The cut rate of the Microsurgical System of 1500-2500 cuts per minute was utilized in all cases.

The infusion line canuula was introduced to inferotemporal cannula. Wide angle fundus visualization was achieved using BIOM noncontact wide field imaging system. Great care was taken to remove all vitreous from around the internal opening of infusion cannula during vitrectomy. Core vitrectomy was performed and the posterior hyaloid was detached and removed in all cases. Peripheral vitreous base shaving with the help of good indentation. 23 gauge pars plana vitrectomy with triamcinolone -assisted posterior vitreous detachment induction. (Kenacort-A; SmithKline Beecham Egypt L.L.C. An affiliated co. to GlaxoSmithKline,) was left standing for 15 minutes and the vehicle of triamcinolone acetonide was discarded. The remaining triamcinolone acetonide $(40 \mathrm{mg})$ suspension was mixed with $5 \mathrm{ml}$ balanced salt solution(BSS) and then was used for the following procedure as a triamcinolone suspension: 
ILM blue (Brilliant blue G) dye was injected under air and left for 30 seconds to one minute ,air fluid exchange were done and the excess dye was removed, a scratch was made in the stained ILM by 23 gauge needle and flap was initiated ,ILM peeling was completed using 23 gauge ILM forceps. Silicone oil (100\% polydimethylsiloxane with a viscosity of 2000 centistock) was used as an internal tamponade in all cases. During removal of the cannulas at the end of the surgery, the eye was pressurized digitally to normal IOP, and the port sites were massaged for few seconds in a direction against that used during trocars insertion. The last step was to check the integrity of the ports to avoid postoperative leakage and suturing of the leaky ports was done when needed.

All patients underwent postoperative positioning with face down position. Posturing was maintained for one week. Silicone oil removal was planned within 2 to 6 months of the initial surgery and was removed in all eyes. Follow up was performed postoperatively at one week, one month, 3 months, and 6 months follow - up .At each visit, best corrected visual acuity (BCVA), intraocular pressure, lens status, intraocular and post ocular complications and anatomical status of the retina were assessed.

\section{Statistical Analysis}

We calculate sample size according to Raosoft and all statistical calculations were done using SPSS (Statistical Package for the Social Science version 20.00; SPSS Inc., Chicago, Illinois, USA). Quantitative data with parametric distribution were done using Analysis of variance $t$ test. The confidence interval was set to $95 \%$ and the margin of error accepted was set to $5 \%$. The p-value was considered non-significant (NS) at the level of $>$ 0.05 , significant at the level of $<0.05,0.01$ and highly significant at the level of $<0.001$. Pearson linear correlation coefficient $(r)$ was estimated to show the relationship between quantitative parameters.

\section{Results}

40 eyes of 31patients (tables :1,2) (Fig.2)underwent pars plana vitrectomy with peeling of internal limiting membrane for diffuse tractional diabetic macular oedema during the study period .The mean age was $60.11 \pm 5$.67years with male to female ratio of 1.00:1.81 (11/40 men $35.5 \%$, and $20 / 40$ female $64.5 \%$ ). The range of the duration of diabetes mellitus(years) in type I (14$35 \mathrm{y})$ and mean $\pm \mathrm{SD} 19.7 \pm 6.183(\mathrm{P}=0.374)$ and the range in Type II (10.000 -40.000y) and mean \pm SD $17.500 \pm 7.811(\mathrm{P}=0.374)$. Distribution of the study group according to the type of diabetes mellitus, where type I( 6 right eye and 4 left eye $25.00 \%$ ) and Type II (12 right eye and 18 left eye $75 \%$ ). The percentage of eyes of patients who had associated systemic conditions among the study group, seven cases had hypertension $17.5 \%$, one case with hypertension and ischemic heart disease $2.5 \%$, one case with renal impairment and anemia2.5\% (two eyes), one case with hepatitis $\mathrm{C}$ virus $2.5 \%$ and one heavy smoker case $2.5 \%$. Preoperative mean visual acuity in logarithm of minimal angle of resolution was (decimal notation) $0.1(6 / 60)(\mathrm{SD} \pm 0.0871)$ ranged from 0.005 to 0.500 with mean of 0.083 in all patients. Mean BCVA(tables:6,7) was 0.01(6/60( Snellens equivalent, meters) which improved after ILM peeling to $0.60(6 / 24$ Snellens equivalent, meters) $(\mathrm{P}<0.05)$ corresponding to 4 line improvement, vision improved to 0.1 ( $\mathrm{SD} \pm 0.098$ ) to 0.3 $(\mathrm{SD} \pm 0.10)$ at 4 weeks to 6 months post-operative. Mean postoperative change in BCVA from base line was $0.0125(\mathrm{SD} \pm 0.071)$ at 4 weeks and $0.043(\mathrm{SD} \pm 0.91)$ at 6 month $(\mathrm{P}=0.003)$. There was a negative correlation number between of pre injection of anti VEGF with mean change of BCVA at 6 months follow up (P $=0.42$ ). Measurements and documentation of preand postoperative intraocular pressure by applanation tonometry revealed an increase of $0.40 \mathrm{mmHg}$ from mean preoperative intraocular pressure of $14.5 \pm 1.45 \mathrm{mmHg} 15.4 \pm 3.5 \mathrm{~mm} \mathrm{Hg}$ at 6 months postoperatively $(\mathrm{P}=0.5)$. Mean preoperative retinal central subfield macular thickness at baseline was $450.71 \mu(\mathrm{SD} \pm 124.067)$. At 6 months there was a median $200 \mu$ decrease from baseline in the central subfield thickness $(\mathrm{P}<0.005)$. Mean change in the central subfield macular thickness was $-150 \mu(\mathrm{SD} \pm 130.56)$. There were $7(17.5 \%)$ (tables :3,4,5)cases developed iatrogenic retinal break during removal of posterior vitreous and treated by endodiathermy, of greatest importance, $3(7.5 \%)$ eyes developed a vitreous hemorrhage treated by conservative treatment after B scan was done, and one eye developed damage to external limiting membrane( fig. 4-8) with marked diminution of vision post- 
operative. All complications were successfully managed. 23 out 40 eyes $(57.5 \%)$ underwent cataract surgery with IOL implantation within 6 months of pars plana vitrectomy. 15 eyes out of 23 cataract surgery $(65.2 \%)$ showed improvement after phacoemulsification and IOL implantation. At 12 months, 5 eyes (12.5\%) received additional argon laser photocoagulation; 3 eyes were injected with combined subtenon's corticosteroids with intravitreal bevacizumab. Median OCT central subfield thickness at 12 months was $240 \mu$ $(\mathrm{SD} \pm 35)$ with the median change from the preoperative OCT measurement being a decrease in thickness of $200 \mu$.

Table (1): Patients'Demographic Data

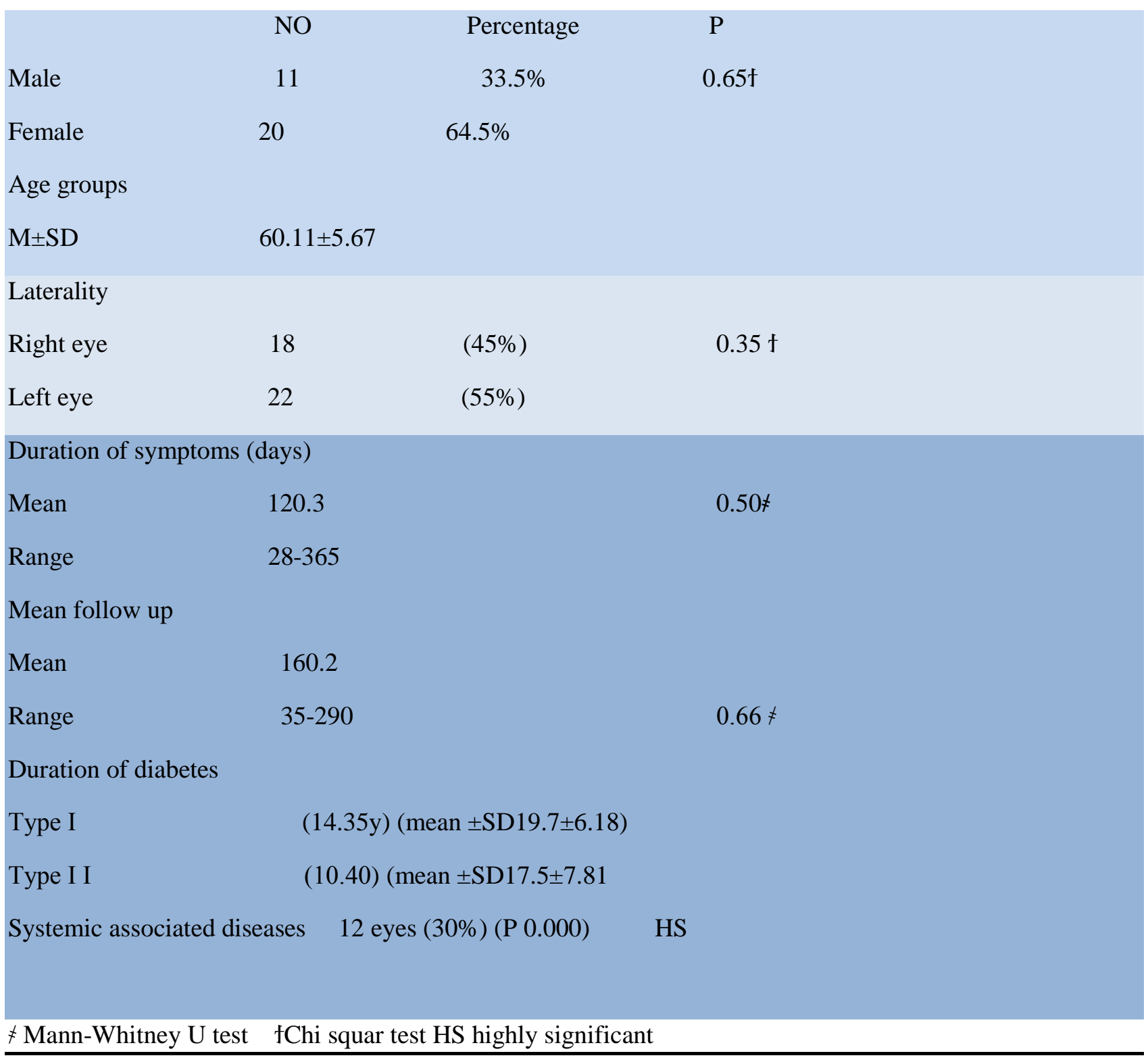


Table (2): Distribution of sex among the study group

\begin{tabular}{|l|l|l|l|l|l|l|}
\hline Gender & \multicolumn{2}{|c|}{ number } & Total & Right & Left \\
\hline Total & N 31 & $\% 100$ & 40 & 22 & 18 \\
\hline Chi -square & $\mathrm{X}^{2}$ & 0.243 & P-value & \multicolumn{2}{|l}{} \\
\hline Male & N 11 & $35.5 \%$ & 15 & 9 & 6 \\
\hline Female & N 20 & $64.5 \%$ & 25 & 13 & 12 \\
\hline
\end{tabular}

Table (3): Number of patients and related complications

\begin{tabular}{|l|l|l|l|}
\hline Number & Single complication & Combined complication & $\%$ \\
\hline 10 & Cataract & & $25 \%$ \\
\hline 4 & & IRB \&cataract & $10 \%$ \\
\hline 1 & Retinal detachment & & \\
\hline 1 & Macular hole & & $2.5 \%$ \\
\hline 1 & ERM & & $2.5 \%$ \\
\hline 2 & Lens touch & & $5 \%$ \\
\hline 1 & Vitreous haemorrhage & & $2.5 \%$ \\
\hline 1 & Glaucoma required surgery & Corneal oedema \& cataract & $2.5 \%$ \\
\hline 1 & & & $2.5 \%$ \\
\hline 3 & Transient IOP & Transient IOP\&IRB & $7.5 \%$ \\
\hline 1 & & Transient IOP\& cataract & $2.5 \%$ \\
\hline 2 & & Lens touch \&IRB & $5 \%$ \\
\hline 2 & & VH\& cataract & $5 \%$ \\
\hline 2 & & IOP \& VH & $5 \%$ \\
\hline 1 & & $2.5 \%$ \\
\hline 23 & Number of lens opacity at 6 months follow up & $52.5 \%$ \\
\hline
\end{tabular}

VH :vitreous haemorrhage ME: macular oedema

IRB: iatrogenic retinal break IOP: intra-ocular pressure

Table (4): Condition of the lens during 6 months follow up

\begin{tabular}{|l|l|l|l|l|}
\hline \multicolumn{5}{|c|}{ Post-operative } \\
\hline & $1^{\text {st }}$ day & One month & 3 month & 6 month \\
\hline Clear lens & $38(95 \%)$ & $25(62.5)$ & $19(47.5)$ & $17(42.5)$ \\
\hline Nuclear & ---- & $10(47.6)$ & $10(47.6)$ & $10(47.6)$ \\
\hline PSC & $2(5 \%)$ & $5(23.8)$ & $5(23.8)$ & $5(23.8)$ \\
\hline Both & & $8(20 \%)$ & $8(20 \%)$ & $8(20 \%)$ \\
\hline
\end{tabular}

This table shows 23 out of 40 clear lens became cataractous.

Table (5): Incidence of complications during operation \& at 6 months follow up

\begin{tabular}{|l|l|l|l|}
\hline Complications & \multicolumn{2}{|c|}{ No } & $\%$ \\
\hline Lens touch & & 3 & $7.5 \%$ \\
\hline \multirow{3}{*}{ New cataract } & Nuclear & 10 & $(47.6)$ \\
\cline { 2 - 4 } & PSC & 5 & $(23.8)$ \\
\cline { 2 - 5 } & both & 8 & $(20 \%)$ \\
\hline
\end{tabular}


Table (6): Visual acuity before and after surgery

\begin{tabular}{|l|l|l|l|l|l|}
\hline Eye number & VA before & VA at 6 month & Eye number & VA before & VA at 6 month \\
\hline 1 & 0.1 & 0.25 & 21 & 0.005 & 0.13 \\
\hline 2 & 0.05 & 0.05 & 22 & 0.005 & 0.2 \\
\hline 3 & 0.2 & 1 & 23 & 0.2 & 0.1 \\
\hline 4 & 0.5 & 1 & 24 & 0.005 & 0.05 \\
\hline 5 & 0.017 & 0.67 & 25 & 0.017 & 0.3 \\
\hline 6 & 0.017 & 0.4 & 26 & 0.033 & 0.3 \\
\hline 7 & 0.033 & 0.2 & 27 & 0.1 & 0.2 \\
\hline 8 & 0.005 & 0.1 & 28 & 0.005 & 0.2 \\
\hline 9 & 0.014 & 0.1 & 29 & 0.033 & 0.2 \\
\hline 10 & 0.014 & 0.1 & 30 & 0.05 & 0.2 \\
\hline 11 & 0.005 & 0.2 & 31 & 0.5 & 0.017 \\
\hline 12 & 0.005 & 0.2 & 32 & 0.05 & 0.2 \\
\hline 13 & 0.017 & 0.4 & 33 & 0.167 & 0.5 \\
\hline 14 & 0.005 & 0.017 & 34 & 0.33 & 0.5 \\
\hline 15 & 0.005 & 0.005 & 35 & 0.014 & 0.033 \\
\hline 16 & 0.2 & 0.017 & 36 & 0.2 & 0.4 \\
\hline 17 & 0.005 & 0.05 & 37 & 0.2 & 0.4 \\
\hline 18 & 0.005 & 0.014 & 38 & 0.01 & 0.2 \\
\hline 19 & 0.005 & 0.4 & 39 & 0.014 & 0.1 \\
\hline 20 & 0.1 & 0.5 & 40 & 0.05 & 0.3 \\
\hline
\end{tabular}
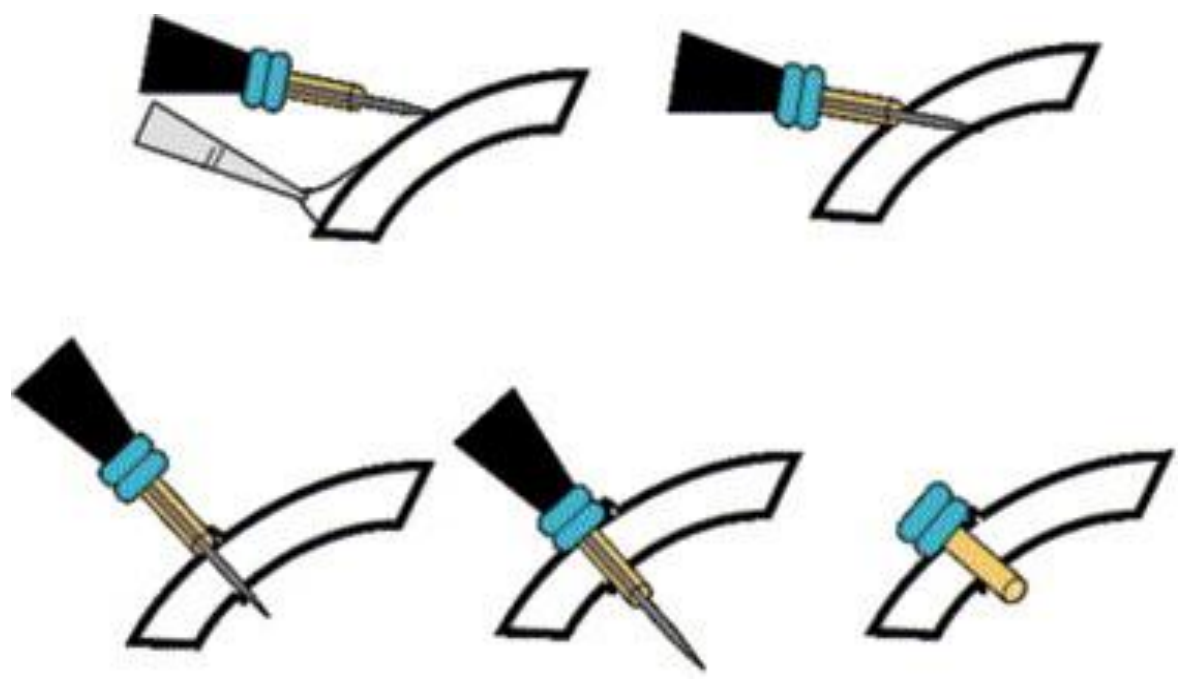

Fig.(1): Steps of sutruless for sclerotomy. (Top left) Conjunctival displacement and beginning of oblique incision. (Top right) End of oblique penetration. (Bottom left) Change of direction of trocar-cannula to perpendicular orientation. (Bottom middle) Perpendicular introduction of trocar-cannula up to cannula collar. (Bottom right) Cannula in place with tilted orientation ${ }^{(18)}$. 
Internal Limiting Membrane Peeling....

Table (7): Visual acuity (Snellen's Equivalent, meters)pre and postoperative at six month

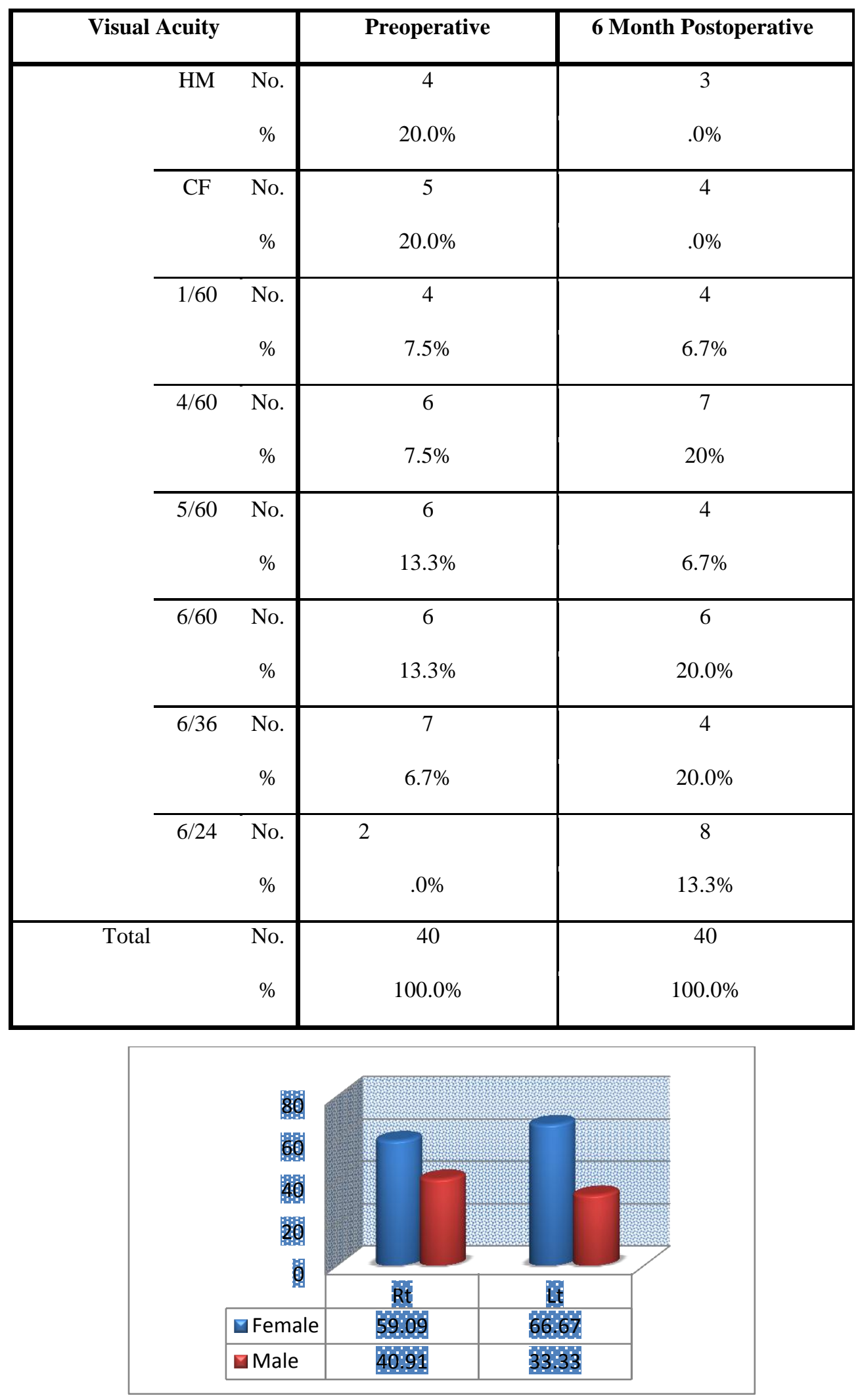

Fig. (2): Distribution of sex among the study group 


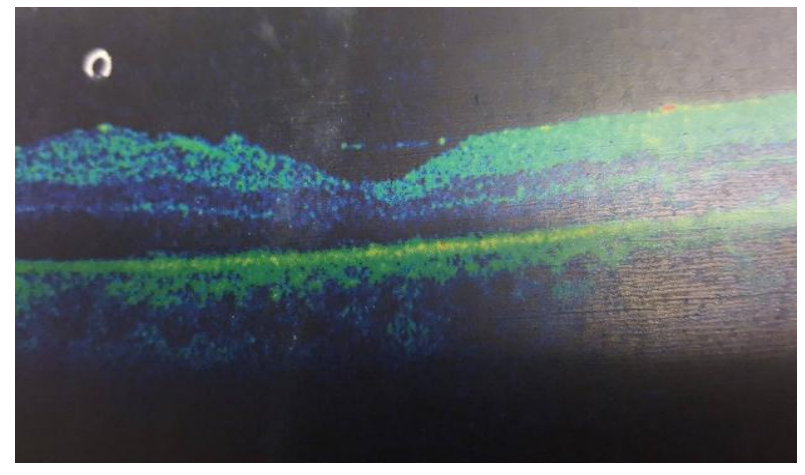

Fig. (3) :PRE retinal fibrosis with focal thickening sparing fovea.

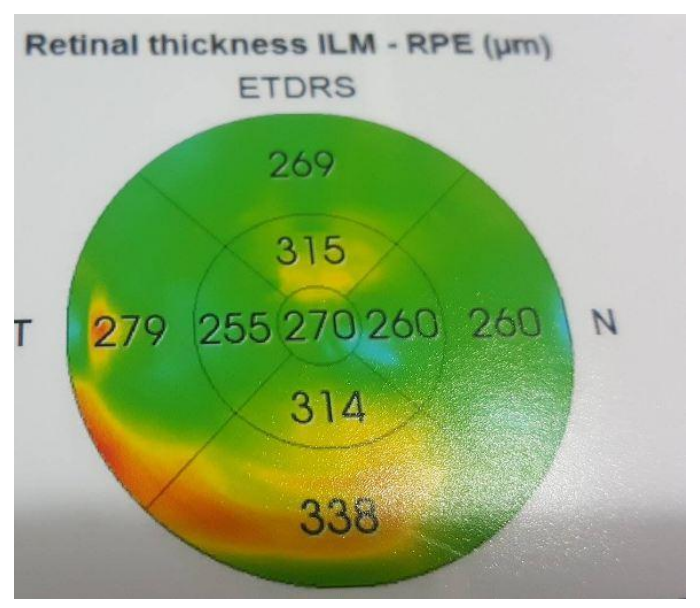

Fig.(4): Macular thickening $270 \mu$

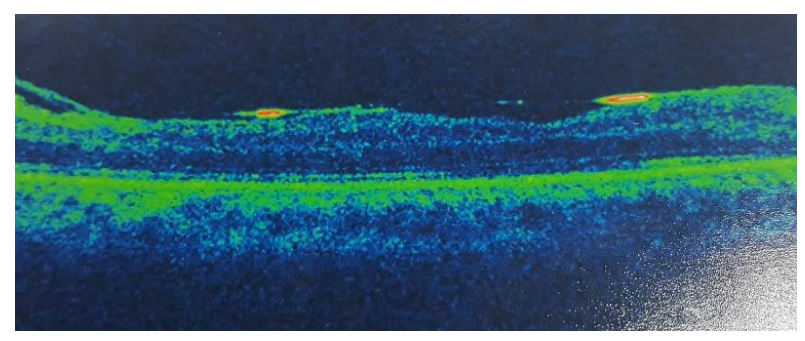

Fig. (5): Eepiretinal Membrane with diffuse macular edema

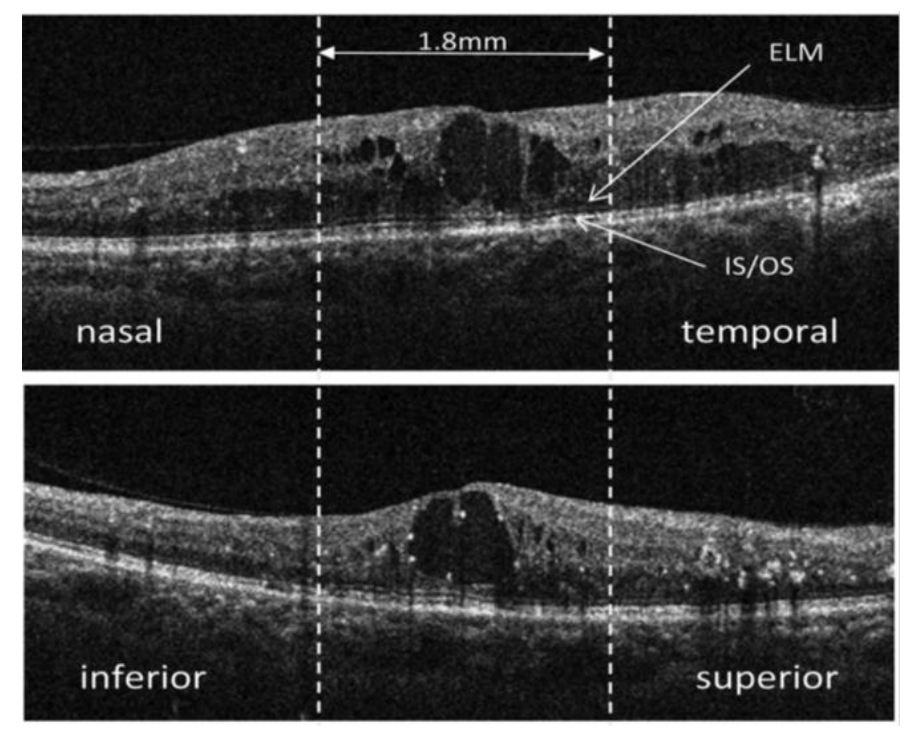

Fig.(6): Diffuse macular oedema

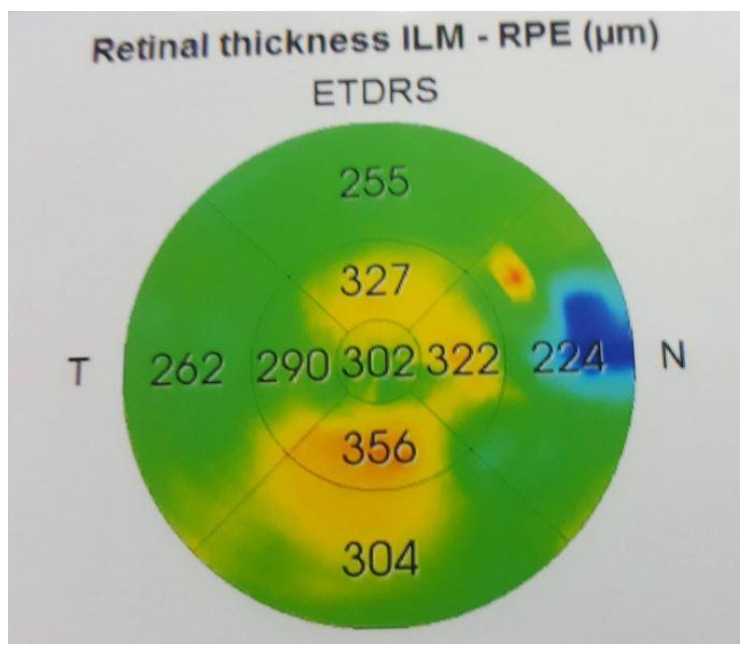

Fig.(7): More worse macular thickening: $302 \mu$

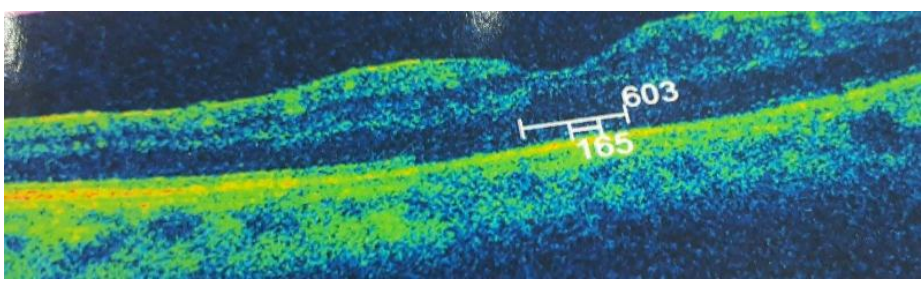

Fig.(8):Macular oedema with focal thicknening involving fovea

ELM \&IS/OS length is 603 micron \& 165 respectively. 


\section{Discussion:}

Tangential and anteroposterior traction on the layers of the macula are considered the primary pathologies behind occurrence of refractory macular oedema .Study considered it in patients who has been treated with focal / grid photocoagulation and persists ${ }^{(5)}$.

In this study 35 eyes (87.5\%) showed marked decrease in mean central macular thickness with marked improvement in macular volume compared to pre - operative values. This anatomical improvement was attributed to surgical removal of the posterior hyaloid which causes a hydrostatic pressure gradient and relieves extravascular leakage and oedma ${ }^{(6)}$. This study was in accordance to Shah et al. ${ }^{(7)}$. Moreover, vitrectomy enhances the intraocular oxygen pressure as it passes from arterial blood into the fluid in the vitreous cavity. Vitrectomy also removes substances that enhance vascular permeability and removal of the posterior hyaloid after injection of the TA which ensure complete removal of cortical vitreous. The anti oedema effect of TA was minor at total drug aspiration during repeated fluid air gas exchange, besides, the half -life of TA was shorter in vitrectomized eye (1.57days) compared to non vitrectomized eye (2.89 days) ${ }^{(8,9)}$. ILM peeling was found to enhance the functional and anatomical outcome by a significant magnitude, even preventing recurrences; thus, clearly establishing the fact that peeling the ILM has a definite advantages ${ }^{(10)}$. One case $(2.5 \%)$ developed ERM during the period of follow up. This result was correlated with the result of Yamamoto et al. ${ }^{(11)}$ who reported 3\% occurrence of ERM after PPV. The preventive effects of ILM peeling are attributed both to complete removal of cellular components on the ILM surface that might develop into an ERM and to removal of the scaffold that promotes cellular proliferation. TA staining also was related to prevention of postoperative $\mathrm{ERM}^{(12)}$. One case $(2.5 \%)$ developed damage to external limiting membrane (Fig.4-8).

Otani et al. ${ }^{(13)}$ performed spectral-domain optical coherence tomography in all eyes. The authors defined central subfield thickness as the average retinal thickness of the $1-\mathrm{mm}$ central scanned area. The length of disruption of the external limiting membrane and the inner and outer segments of the photoreceptors in the fovea (1.8 $\mathrm{mm}$ in diameter) were measured and graded according to their length as follows: 1) $>1.4 \mathrm{~mm}$; 2) $>0.4 \mathrm{~mm}$ but $<1.4 \mathrm{~mm}$; or 3$)<0.4 \mathrm{~mm}$. Spectral-domain optical coherence tomography showed that the integrity of the external limiting membrane and inner and outer segments of the photoreceptors was more strongly correlated with best-corrected visual acuity when compared with central subfield thickness in diabetic macular oedema ${ }^{(13)}$.

Preoperative visual acuity in logarithm of minimal angle of resolution was (decimal notation) $0.1(6 / 60)(\mathrm{SD} \pm 0.0871)$ ranged from 0.005 to 0.500 with mean of 0.083 in all patients. Mean BCVA was 1.01(6/60 Snellen's equivalent) which improved after ILM peeling to $0.60(6 / 24$ Snellen's equivalent) $(\mathrm{P}<0.05)$ corresponding to 4 line improvement, vision improved to 0.1 ( $\mathrm{SD} \pm$ $0.098)$ to $0.3(\mathrm{SD} \pm 0.10)$ at 4 weeks to 6 months post operatively. Mean postoperatively change in BCVA from base line was $0.0125(\mathrm{SD} \pm 0.071)$ at 4 weeks and $0.043(\mathrm{SD} \pm 0.91)$ at 6 month (P0.003) .It was also found that visual improvement at 3 months post-operatively was positive $0.6(\mathrm{P}<$ $0.05)$. Thomson et al.$^{(\mathbf{1 4})}$ defined six preoperative and three intra operative factors were found to be associated with an improved likely hood of attaining a final visual acuity of $5 / 200$ or better. The preoperative factors were as follows: preoperative visual acuity of $6 / 60$ or better, the absence of iris neovascularization, a clear crystalline lens or only a minimal cataract, minimal or no preoperative vitreous hemorrhage, PRP of at least one fourth of the retina, and the absence of severe retinal neovascularization. The intraoperative factors were as follows: the avoidance of performing a lensectomy, the absence of an iatrogenic retinal break, and the avoidance of using intraocular gas. In current study; improvement in mean BCVA agreement with Stolba et al. ${ }^{(15)}$. Pendergast et al. ${ }^{(16)}$ the most common postoperative complication after vitrectomy was cataract formation, which occurred in $24(63.2 \%)$ of the 38 phakic eyes. In our study, cataract occurred 23 out 40 eyes $(57.5 .5 \%)$ underwent cataract surgery with IOL implantation within 6 months of pars plana vitrectomy9 out of $15(60 \%)$ patients showed improvement. Phakic eyes with silicone oil internal tamponade (16 cases, 40\%) ${ }^{(16)}$.The 
incidence of postoperative cataract formation in our study was closely related to other researchers. Cataract extraction with IOL implantation was done during silicone oil removal at least four months after surgery. Glaucoma was described by many authors in varying percentages (from $1.7 \%$ to $8 \%$ ) after vitrectomy ${ }^{(17)}$.

Transient increase in IOP was realized in 3 eyes (7.5\%). Medical treatment in the form of IOP lowering drops was successful in managing those cases without need for glaucoma filtering surgery. In our study glaucoma did not affect the final visual outcome of our patients. The intraoperative and postoperative complications of the surgery in this study were few and did not affect the final visual outcome.

In conclusion, ILM peeling achieved higher anatomic success with a reduced need for additional surgical interventions and or event postoperative ERM formation that might result in subsequent visual loss.

Compliance with ethical standards.

Funding No funding was received for this research.

Conflict of interest: certifies that he has no affiliations with or involvement in any organization or entity with any financial interest in the subject matter or materials discussed in this manuscript.

\section{References:}

1. Chai L and Morris JE (1999): Heparin sulfate in the inner limiting membrane of embryonic chicken retina binds basic fibroblast growth factor to promote axonal outgrowth. Exp Neurol., 1601:175-185.

2. Sakamoto $\mathbf{T}$ and Ishibashi $\mathbf{T}(\mathbf{2 0 1 1})$ : Hyalocytes: essential cells of the vitreous cavity in vitreoretinal pathophysiology. Retina ,31:222-228.

3. Tadavoni R, Svorenova I, Erginary A et al.(2012) :Decreased retinal sensitivity after internal limiting membranes peeling for macular hole surgery. $\mathrm{Br} \mathrm{sJ}$ Ophthalmol., 96:1513-1516.

4. Gelman R, Steven W, Prospero Ponce C et al. (2015): Retinal Damage Induced by Internal Limiting Membrane
Removal, J of Ophthalmology vol. 2015, Article ID 939748, http://dx.doi.org/10.1155/2015/939748.

5. Patel JI, Hkin PG, Schadt $M$ et al. (2006): Pars plana vitrectomy for diabetic macular oedema :OCT and functional correlations. Eye 20,674-680.

6. Tachi $N$ and Ogino N(1994): Vitrectomy for diffuse macular oedema associated with diabetic retinopathy. J eye , 1077-1081.

7. Shah SP, Patel M, Thomas D et al. (2006): Factors predicting outcome of vitrectomy for diabetic macular oedema: results of a prospective study. $\mathrm{Br} \mathrm{J}$ Ophthalmol., 90:33-36.

8. Yamamoto $T$, Yamamoto $S$ and Takeuchi S(2000): Pars plana vitrectomy for diabetic macular oedema with posterior vitreous detachment .J Eye ,17:133-138.

9. Chin HS, Park TS, Moon YS et al. (2005): difference in clearance of intravitreal triamcinolone acetonid between vitrectomied and non vitrectomized eyes. Retina , 5:556-560.

10. Brooks HL (2000): Macular surgery with and without internal limiting membrane peeling. Ophthalmology, 107:1939-1949.

11. Yamamoto T, Akahane $\mathbf{N}$, Takeuchi $S$ (2001): Vitrectomy for diabetic macular edema: the role of posterior vitreous detachment and epimacular membrane. Am J Ophthalmol., 132(3):369-377.

12. Akiyama K, Fujinami $K$, Watanabe $K$ et al. (2016) :Internal limiting membrane peeling to prevent post vitrectomy epiretinal membrane development in retinal detachment .Am J Ophthalmol., 171:1-10.

13. Otani T, YamaquchiY, Kishi S et al. (2010): correlation between visual acuity and foveal microstructural changes in diabetic macular oedema .Retina , 30(5) :774-780.

14. Thompson JT, Glaser BM, Michels RG et al.(1986): The use of intravitreal thrombin to control hemorrhage during vitrectomy. Ophthalmology , 93:279282. 
15. Stolba U, Binder $S$ and Gruber D et al. (2000): Vitrectomy for persistent diffuse diabetic macular oedema associated with premacular posterior hyaloid .Am J Ophthalmol. ,130:178-86.

16. Pendregast SD,Hassan TS , Williams GA et al.(2000): Vitrectomy for diffuse diabetic macular edema associated with a taut premacular posterior hyaloid. Am J Ophthalmol .,130: 178- 186.
17. Yang CM (2000): Surgical treatment for sever diabetic macular edema with massive hard exudates. Retina, 20(2): 121- 125.

18. Lorenzo LG, Jeslis PE and Miguel AT(2006): Oblique Sclerotomy Technique for Prevention of Incompetent Wound Cosure in Transconjunctival 25Gauge Vitrectomy. Am J Ophthalmol., 141: 1154-1156 